\title{
SISTEMAS METEOROLÓGICOS ASSOCIADOS À OCORRÊNCIA DE PRECIPITAÇÃO NO CENTRO DE LANÇAMENTO DE ALCÂNTARA
}

\author{
SHEILA SANTANA DE BARROS ${ }^{1}$, MARCOS DAISUKE OYAMA ${ }^{2}$ \\ ${ }^{1}$ Instituto Nacional de Pesquisas Espaciais/Centro de Previsão de Tempo e Estudos Climáticos (INPE/ \\ CPTEC), São José dos Campos, SP, Brasil \\ ${ }^{2}$ Centro Técnico Aeroespacial/Instituto de Aeronáutica e Espaço (CTA/IAE), \\ São José dos Campos, SP, Brasil \\ sheila.santana@cptec.inpe.br,oyama@iae.cta.br
}

Recebido Novembro 2008 - Aceito Dezembro 2009

\begin{abstract}
RESUMO
O Centro de Lançamento de Alcântara (CLA; $\left.2^{\circ} 22^{\prime} \mathrm{S}, 44^{\circ} 23^{\prime} \mathrm{W}\right)$ tem papel importante para as atividades aeroespaciais no Brasil, sendo de interesse estudar os sistemas precipitantes atuantes sobre a região. O trabalho teve como objetivo caracterizar os sistemas associados aos eventos de precipitação ocorridos no CLA entre 2005 e 2006, utilizando totais horários de precipitação, dados de Reanálise do NCEP/NCAR, temperatura de brilho do satélite GOES-12 e radiação de onda longa emergente. Para tal, definiram-se critérios para identificar os sistemas meteorológicos de grande, meso e de escala local associados à precipitação no CLA. Os resultados apontaram que $40 \%$ dos eventos de precipitação estiveram associados a fatores de grande escala, destacando-se a Zona de Convergência Intertropical. Verificou-se que 60\% (40\%) dos eventos de precipitação se devem a processos estratiformes (convectivos). Os sistemas convectivos apresentaram as seguintes características: áreas entre $10^{4} \mathrm{~km}^{2}$ e $25 \times 10^{4} \mathrm{~km}^{2}$, chuva posicionada na porção convectiva do sistema, intensificação por convecção diurna, inclusão em um sistema de escala maior e origem preferencial de sudeste ou nordeste. Os sistemas formados por processos estratiformes se subdividiram quase igualmente em dois grupos: resquícios de sistemas convectivos anteriormente atuantes no CLA e sistemas que não estão associados a nenhum processo convectivo.
\end{abstract}

Palavras-chaves: chuva, sistemas convectivos, costa norte do Brasil

\begin{abstract}
RAINFALL AT THE ALCÂNTARA ROCKET LAUNCHING CENTER: OBSERVATIONAL AND MODELING ASPECTS

The Alcântara Rocket Launching Center (CLA; $2^{\circ} 22^{\prime} \mathrm{S}, 44^{\circ} 23^{\prime} \mathrm{W}$ ) plays an important role in aerospace research activities in Brazil, and so it is of interest to study the precipitation systems over the CLA region. This work aimed at characterizing the systems associated with precipitation occurrence at CLA from 2005 to 2006 by using hourly precipitation data, NCEP/NCAR Reanalysis data, brightness temperature data from GOES-12 satellite and outgoing longwave radiation data. Criteria were defined to identify large, meso and local scale meteorological systems associated with precipitation at CLA. About $40 \%$ of precipitation events were associated with large scale systems, mainly the Intertropical Convergence Zone, and about $60 \%$ (40\%) of precipitation events are due to convective (stratiform) processes. The convective systems showed the following characteristics: area between $10^{4} \mathrm{~km}^{2}$ e $25 \times 10^{4} \mathrm{~km}^{2}$, precipitation located in convective part of the system, intensification by diurnal convection, inclusion in a larger scale system and preferred southeasterly or northeasterly propagation. The systems formed from stratiform processes could be divided almost equally in two groups: reminder of convective systems previously active over CLA and systems that are not associated with any convective process.
\end{abstract}

Keywords: rainfall, convective systems, Brazilian northern coast 


\section{INTRODUÇÃO}

A região tropical do globo é influenciada por diversos sistemas meteorológicos que atuam em diferentes escalas espaciais, e podem levar à ocorrência de precipitação. Em específico na região norte do nordeste brasileiro (NNEB), os sistemas que atuam em escala sinótica são Zona de Convergência Intertropical (ZCIT), Vórtice Ciclônico em Altos Níveis (VCAN), Distúrbios Ondulatórios de Leste (DOL), Zona de Convergência do Atlântico Sul (ZCAS) e Sistemas Frontais (SF); em mesoescala, Linhas de Instabilidade (LI) e Sistemas Convectivos de Mesoescala (SCM); e em escala local, tempestades isoladas e circulação de brisa (Cavalcanti, 1982; Cohen et al., 1989; Molion e Bernardo, 2002; Tavares, 2008).

Este trabalho foca uma região específica do NNEB: a região do Centro de Lançamento de Alcântara (CLA, Alcântara, MA; $2^{\circ} 22^{\prime} \mathrm{S}, 44^{\circ} 23^{\prime} \mathrm{W}$; Marques e Fisch, 2005), localizado no litoral do Maranhão. No CLA, realiza-se o lançamento de foguetes projetados e construídos no Instituto de Aeronáutica e Espaço (IAE). O lançamento de um foguete requer informações detalhadas de elementos meteorológicos para que os requisitos da Segurança de Vôo sejam atendidos no momento do lançamento. Neste sentido, uma pesquisa que detalhe os sistemas meteorológicos associados à precipitação atuantes na região do CLA é de interesse aeroespacial.
Algumas informações meteorológicas como dados de superfície, ar superior e satélite (no período de 1993 a 1999) foram utilizadas por Pereira et al. (2002) para obter as principais características climatológicas da região que abrange o CLA. Foi observado que a estação chuvosa (seca) ocorre entre os meses de janeiro e junho (julho e dezembro), e os máximos (mínimos) de precipitação ocorrem nos meses março e abril (setembro e novembro). Nos meses chuvosos (secos), a cobertura média de nuvens é maior (menor) e os ventos são menos (mais) intensos. $\mathrm{O}$ mês de novembro (março) apresenta temperatura média mensal maior (menor) do que a de outros meses, $\operatorname{com} 28,1^{\circ} \mathrm{C}$ $\left(26,7^{\circ} \mathrm{C}\right)$; assim, a variação sazonal da temperatura média mensal é pequena. No ciclo diurno, a cobertura de nuvens é maior (menor) entre 07 e $12 \mathrm{HL}$ ( 21 e $02 \mathrm{HL}$ ), os ventos mais (menos) intensos ocorrem no período diurno (noturno), e a temperatura máxima (mínima) ocorre entre 12 e 14 HL (05 e $06 \mathrm{HL})$

Utilizando dados horários de precipitação CLA entre 1993 e 2007, Barros (2008) realizou uma caracterização estatística dos eventos de precipitação. Na estação chuvosa (seca) do CLA, o número médio de eventos por mês encontra-se entre 50 e 60 (é menor que 10). Em geral, os eventos de chuva possuem pouco total acumulado $(<0,5 \mathrm{~mm})$, curta duração $(<1$ h), pequena intensidade média $\left(<0,5 \mathrm{~mm} \mathrm{~h}^{-1}\right)$ e são freqüentes (muitas vezes ao dia) na estação chuvosa. O número de eventos de chuva, em DJF, é uniformemente distribuído durante o dia;

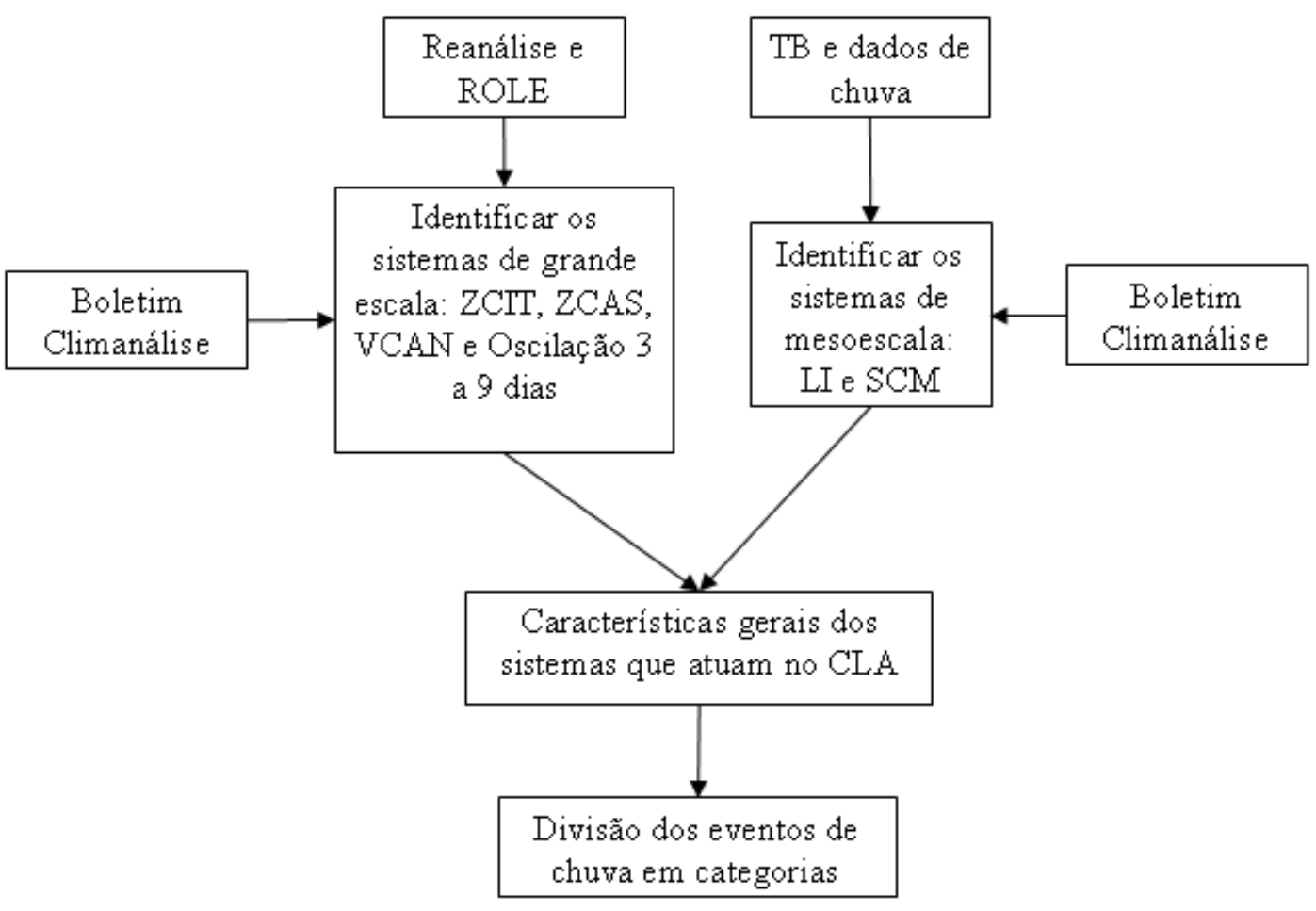

Figura 1 - Fluxograma das etapas do trabalho. 
em MAM, apresenta um máximo entre 03 e 09 HL; em JJA, apresenta um máximo entre 03 e $06 \mathrm{HL}$; e em SON, concentra-se no período diurno. $\mathrm{O}$ total de precipitação, em DJF, apresenta um máximo entre 18 e 06 HL; em MAM e JJA, entre 18 e 03 HL; e em SON, entre 18 e $21 \mathrm{HL}$.

O objetivo deste trabalho é caracterizar os sistemas meteorológicos associados a eventos de precipitação no CLA nos anos de 2005 e 2006. Para tal, analisam-se as características da nebulosidade (utilizando dados de satélite) e a atuação de sistemas de grande escala (utilizando dados de Reanálise e informações do Boletim Climanálise), durante os eventos de precipitação ocorridos em 2005 e 2006 (identificados de dados de superfície). Procura-se responder às seguintes questões: quais são os principais fatores (sinóticos, de mesoescala ou locais) associados à precipitação no CLA?; e quais são as principais características destes sistemas?

\section{DADOS E METODOLOGIA}

Os seguintes materiais, para o período de 2005 a 2006, são utilizados:

- Dados de total horário de precipitação no CLA disponibilizados pela Divisão de Ciências Atmosféricas (ACA) do IAE;

- Dados de temperatura de brilho (TB) no canal infravermelho do satélite GOES-12 com espaçamento temporal de 30 minutos e horizontal de $4 \mathrm{~km}$, disponibilizados pela DSA (Divisão de Satélites e Sistemas Ambientais) do CPTEC;

- Dados de Reanálise do NCEP/NCAR (Kalnay et al., 1996) com espaçamento horizontal de $2,5^{\circ}$ e temporal de $6 \mathrm{~h}$, obtidos do sítio ${ }^{1}$ da internet do "Climate Diagnostics Center" / "National Oceanic and Atmospheric Administration" (CDC/NOAA);

- Dados de radiação de onda longa emergente (ROLE) diária com espaçamento horizontal de $2,5^{\circ}$, obtidos do sítio ${ }^{2}$ da internet do CDC/NOAA;

- Boletim Climanálise, editado mensalmente pelo CPTEC/INPE.

As etapas do trabalho e os dados utilizados em cada etapa estão mostrados na Figura 1. A metodologia baseia-se na identificação dos fatores/sistemas meteorológicos atuantes no CLA e adjacências, assim como a caracterização da nebulosidade associada, ambos nos horários de ocorrência de chuva.

São avaliados os seguintes fatores sinóticos: Zona de Convergência Intertropical (ZCIT), Vórtice Ciclônico de Altos Níveis (VCAN), Oscilação de 3 a 9 dias e Zona de Convergência do Atlântico Sul (ZCAS). Os seguintes procedimentos são utilizados para selecionar quais destes fatores estavam associados com precipitação do CLA:

A seleção das pêntadas em que a ZCIT atuou como causadora de chuvas no CLA, obedece aos seguintes critérios (Marques e Baungartner, 2008; Ferreira et al., 2005): ROLE menor que $220 \mathrm{~W} \mathrm{~m}^{-2}$, convergência atmosférica em $850 \mathrm{hPa}$ menor que $-4,5 \times 10^{-6} \mathrm{~s}^{-1}$ e posição abaixo do Equador.

Casos de VCAN são identificados se o campo de linha de corrente em $200 \mathrm{hPa}$ indicar uma circulação ciclônica fechada com vorticidade relativa negativa, e a distância do CLA à borda do vórtice (DAV) menor do que $2000 \mathrm{~km}$ (Silva, 2005).

Os dias em que a Oscilação de 3 a 9 dias esteve ativa são selecionados a partir de um filtro passa-banda (média móvel) da componente meridional do vento em $700 \mathrm{hPa}$ para reter

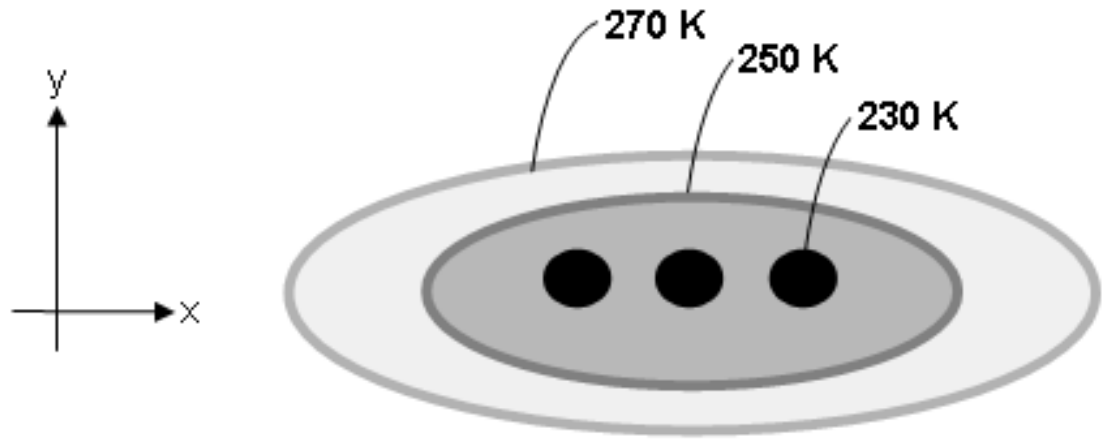

Figura 2 - Ilustração dos limiares adotados para a separação da nebulosidade convectiva da estratiforme em um corte na horizontal. A nebulosidade estratiforme é identificada através de $\mathrm{TB}<270 \mathrm{~K}$, a nebulosidade convectiva é identificada por $\mathrm{TB}<250 \mathrm{~K}$ e os núcleos convectivos por $\mathbf{T B}<\mathbf{2 3 0} \mathbf{K}$.

\footnotetext{
${ }^{1} \mathrm{http}: / /$ www.cdc.noaa.gov/cdc/data.ncep.reanalysis.html

${ }^{2} \mathrm{http}: / /$ www.cdc.noaa.gov/cdc/data.interp_OLR.html
} 
as oscilações de 3 a 9 dias. Selecionam-se os casos em que a amplitude do vento filtrado seja maior que $0,5 \mathrm{~m} \mathrm{~s}^{-1}$ e tal que a componente do vento mude de sinal (de positivo para negativo) na longitude do CLA (Alves et al., 2008).

Os dias em que a ZCAS esteve ativa são selecionados a partir do Boletim Climanálise (Climanálise, 2005, 2006).

Ao final, obtém-se uma lista diária com a informação de qual sistema esteve atuando na região do CLA.

A partir de imagens de temperatura de brilho (TB) e informações do Boletim Climanálise, são observados os padrões

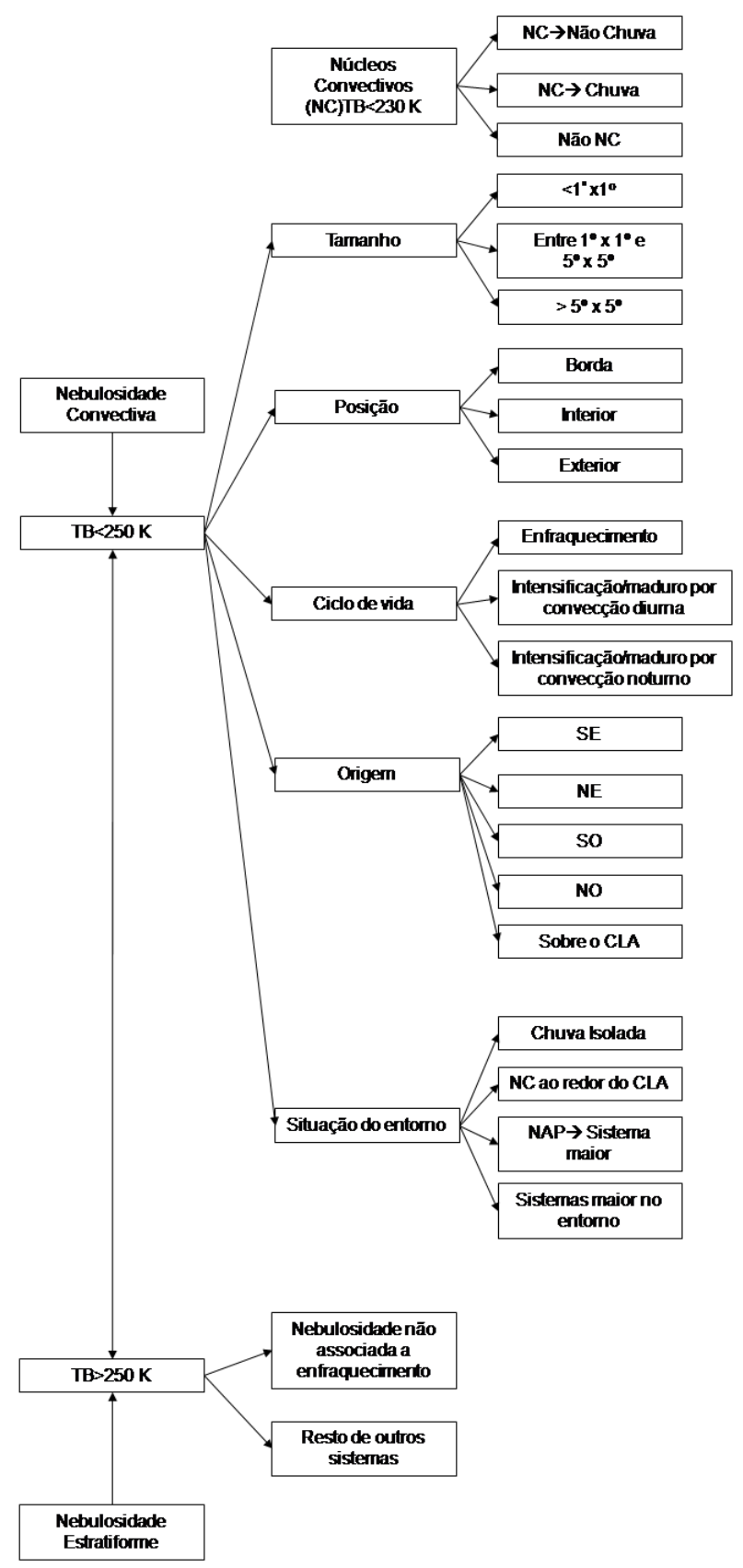

Figura 3 - Esquema da caracterização dos sistemas. de nebulosidade associados à precipitação em todos os eventos dos anos de 2005 e 2006. O limiar de $250 \mathrm{~K}$ é utilizado para identificar sistemas convectivos ( $\mathrm{SC}$; TB $>250 \mathrm{~K}$ ). Um limiar bem próximo a esse (TB $<245 \mathrm{~K}$ ) foi utilizado por Vila e Machado (2004) para identificar nuvens associadas à convecção.

Linhas de Instabilidade (LI) e Sistemas Convectivos de Mesoescala (SCM) são identificados da seguinte forma:

As LI são identificadas subjetivamente através de dados de TB às 21 UTC, horário no qual as LI encontram-se bem definidas (Cohen et al., 1989), e de acordo com os padrões clássicos de LI descritos em Cavalcanti (1982);

Os SCM são identificados através de dados de TB, verificando se a nebulosidade possui um padrão circular (Maddox, 1980) e tamanho horizontal suficientemente grande, i.e., área mínima (do SC, i.e., TB < 250 K) de $10^{4} \mathrm{~km}($ Cf. Orlanski, 1975).

A Figura 2 ilustra os limiares adotados - além do limiar de $250 \mathrm{~K}$, utilizam-se os limiares de $270 \mathrm{~K}$ (nebulosidade estratiforme entre 250 e $270 \mathrm{~K}$ ) e $230 \mathrm{~K}$ (núcleos convectivos para $\mathrm{TB}<230 \mathrm{~K}$ ) - para identificar e caracterizar a nebulosidade associada à precipitação no CLA (NAP). A divisão dos eventos associados à nebulosidade convectiva e estratiforme é feita da seguinte forma (Figura 3):

Se a NAP não está associada a um SC (TB > $250 \mathrm{~K})$, os eventos são subdivididos em dois grupos: o primeiro (segundo) contém os eventos que não estão associados (estão associados) a enfraquecimento de SC.

Se a NAP está associada a um SC (TB $<250 \mathrm{~K})$, algumas características mais específicas são analisadas:

a) Existência de núcleos convectivos (TB < $230 \mathrm{~K}$ ). Os sistemas podem possuir núcleos convectivos no seu interior, i.e., aglomerado de nuvens com TB abaixo de $230 \mathrm{~K}$. Os eventos possuem três configurações básicas que estão ilustradas na Figura 4a-c e são subdivididas em três grupos: (1) não há núcleos convectivos no interior da área onde TB $<250 \mathrm{~K}$ (Figura 4a); (2) há núcleos convectivos, mas nenhum deles encontra-se sobre o CLA (Figura 4b); e (3) há núcleo convectivo sobre o CLA (Figura 4c).

b) Área do sistema. Os eventos estão divididos em três grupos em função da área do sistema com $\mathrm{TB}<250 \mathrm{~K}$. No primeiro, encontram-se os eventos cujo sistema possui área menor que $1^{\circ} \times 1^{\circ}\left(\sim 10^{4} \mathrm{~km}^{2}\right)$; no segundo, área entre $1^{\circ} \times 1^{\circ} \mathrm{e}$ $5^{\circ} \times 5^{\circ}\left(\sim 10^{4} \mathrm{~km}^{2}\right.$ a $\left.25 \times 10^{4} \mathrm{~km}^{2}\right)$; no terceiro, área superior a $5^{\circ} \times 5^{\circ}\left(\sim 25 \times 10^{4} \mathrm{~km}^{2}\right)$. As perturbações convectivas definidas em Guedes e Machado (2003) possuem tamanhos no limite entre o segundo e terceiro grupos (escala meso $\alpha$ conforme Orlanski, 1975).

c) Posição do sistema em relação ao CLA. No primeiro grupo selecionam-se os eventos nos quais a borda do sistema encontra-se sobre o CLA (Figura 4d). No segundo grupo, o CLA está no interior do sistema (Figura 4e). No terceiro grupo (Figura 
4f), o CLA permanece no exterior do sistema (i.e., sobre o CLA, $\mathrm{TB}>250 \mathrm{~K}$, mas a NAP estaria associada à parte estratiforme de um sistema com $\mathrm{TB}<250 \mathrm{~K}$ próximo do CLA).

d) Fase do ciclo de vida. Esta característica também é dividida em três novos grupos: (1) eventos cujo sistema está na fase final do ciclo de vida, i.e., no enfraquecimento ou na dissipação, (2) eventos cujo sistema apresenta intensificação ou está maduro por convecção diurna e (3) eventos cujo sistema apresenta intensificação ou está maduro por convecção noturna.

e) Situação do entorno. Esta caracterização é realizada para avaliar como se apresenta o entorno do CLA no momento da precipitação. A Figura 4g-j representa algumas das possíveis situações de entorno visualizadas durante o período de estudo.
Para tal, são estabelecidos quatro novos grupos: (1) eventos considerados como chuva isolada, i.e., o entorno apresenta apenas um sistema de tamanho pequeno sobre o CLA e nenhum outro sistema no entorno (Figura 4g), (2) eventos que apresentam SC isolados ao redor do CLA (Figura 4h), (3) eventos cujo sistema está incluso em um outro sistema de maior escala (Figura 4i) e (4) eventos que possuem um sistema de maior escala no entorno do CLA, mas esse sistema não engloba a NAP (Figura 4j).

f) Origem do sistema. O sistema é acompanhado (tracking) subjetivamente, sempre que possível, desde sua origem. Os eventos são subdivididos em relação ao local de origem do sistema: nordeste, noroeste, sudeste e sudoeste do CLA ou sobre o CLA (Figura 4k).

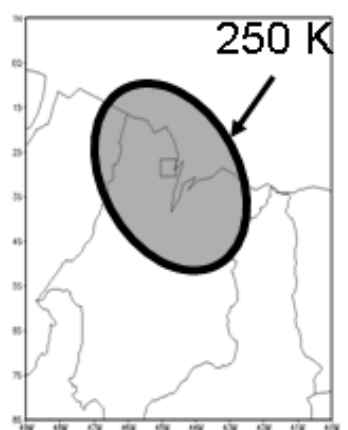

(a)

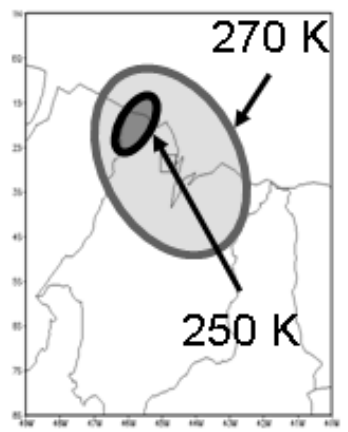

(e)

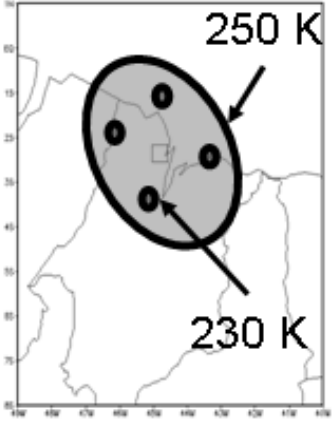

(b)

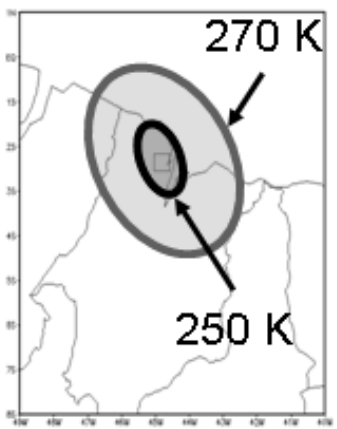

(f)

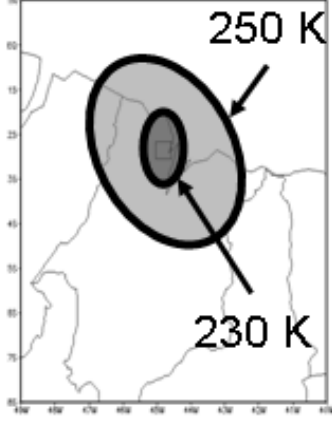

(c)

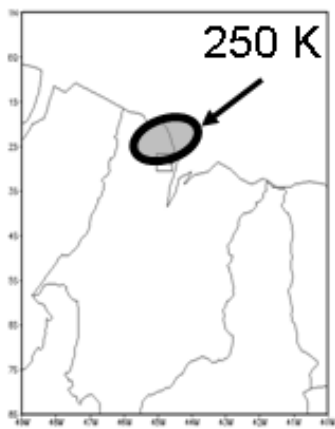

(g)

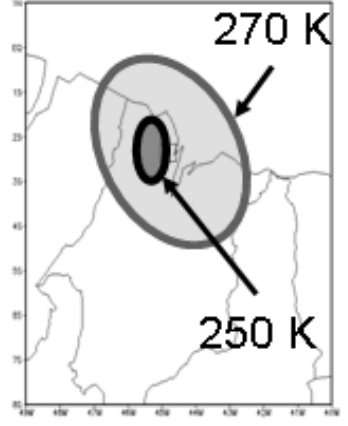

(d)

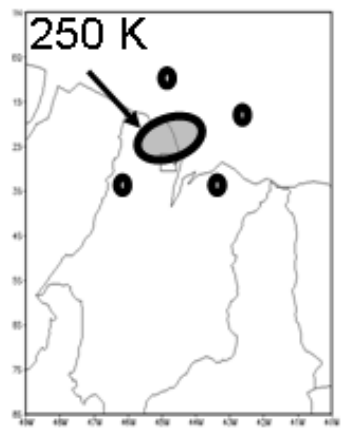

(h)

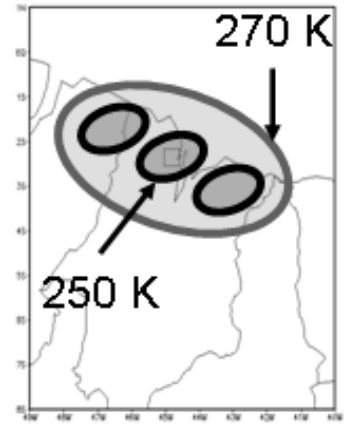

(i)

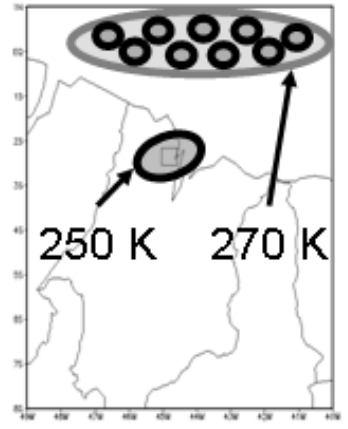

(j)

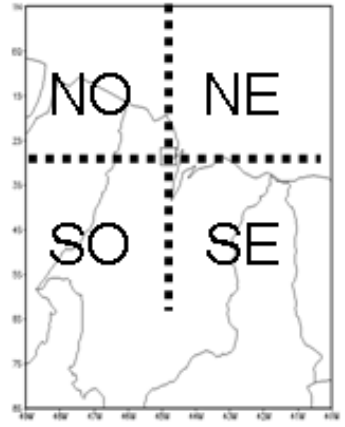

(k)

Figura 4 - Esquema conceitual que representa: (a), (b) e (c) disposição dos núcleos convectivos; (d), (e) e (f) posição da nebulosidade; (g), (h), (i) e (j) situação do entorno e (k) região de origem. 
Calculam-se o número de eventos e o total de precipitação associados a cada uma das características descritas acima, além das suas respectivas subdivisões. Para cada uma das características, são excluídos os eventos para os quais não é possível obter alguma característica (p.ex., por falta de imagens de satélite). Por exemplo, a origem do sistema (TB $<250 \mathrm{~K}$ ) pode estar em um sistema de grande escala e, por isso, torna-se difícil decidir a partir de qual região o sistema se inicia. Por isso, são considerados somente os eventos em que é possível realizar a análise e este total será denominado total de eventos.

\section{RESULTADOS E DISCUSSÃO}

A Figura 5 mostra a contribuição relativa dos fatores de grande, meso e pequena escala para os eventos de precipitação ocorridos no CLA em 2005 e 2006. Os fatores de grande escala isoladamente (i.e., sem ação conjunta de fatores de mesoescala ou escala local) são os responsáveis por cerca de $1 / 3$ dos casos de precipitação do CLA nos dois anos. Além de atuar isoladamente, os fatores de grande escala interagiram com os de mesoescala em uma grande fração de casos $(26 \%$ em 2005 e 57\% em 2006). Assim, conclui-se que os fatores de grande escala constituem-se no principal causador de chuva no CLA (isoladamente ou associados a fatores de mesoescala). Os processos em escala local e os sistemas de mesoescala atuando sem a presença de fatores de grande escala, estão associados a uma menor porcentagem dos casos de precipitação ocorridos no CLA. Embora a fração de casos associados a sistemas locais seja relativamente pequena, na ordem de $10 \%$, este número corresponde a mais do que uma dezena de casos e pode, dessa forma, influenciar a previsibilidade de precipitação no CLA.

A Tabela 1 mostra a porcentagem do total de eventos associado a cada sistema meteorológico considerado neste trabalho. As colunas de 2005 e 2006 somam mais do que $100 \%$; isto ocorre, pois existem de eventos que estão associados simultaneamente a mais do que um sistema. O principal sistema meteorológico associado aos eventos de chuva no CLA é a ZCIT, com mais da metade do total de eventos nos dois anos. Este resultado concorda com Molion e Bernardo (2002), que

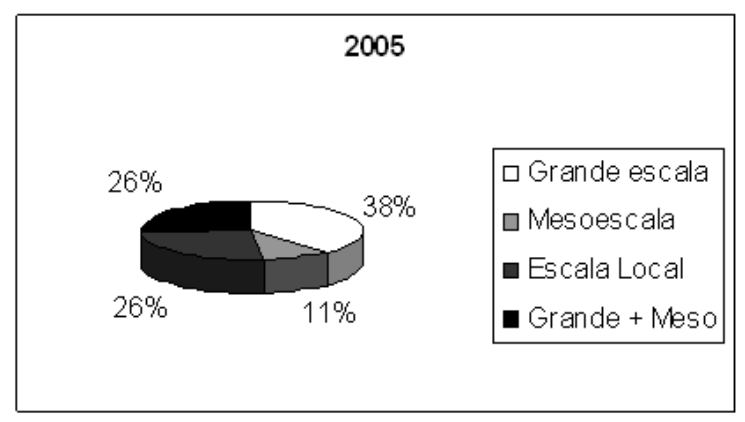

consideram a ZCIT como o principal causador de chuvas na região. Outros sistemas que se destacaram pela quantidade de eventos de chuva associados foram as LI (na ordem de 1/4 a 1/3) e os SCM (na ordem de 1/4). Os sistemas que apresentam o menor número de casos de precipitação associados foram VCAN e Oscilação de 3 a 9 dias.

Os sistemas dificilmente atuam individualmente e por isso foi realizada uma análise da associação entre os sistemas, que pode ser vista nas Tabelas 2 e 3. A diagonal das tabelas (células sombreadas) mostra o número de casos dos sistemas que se apresentam individualmente. A ZCIT é o sistema com maior freqüência de aparecimento individual (Tabela 2). Os sistemas de grande escala que mais estiveram associados à ZCIT foram ZCAS e VCAN. Observa-se uma menor freqüência de sistemas de grande escala associados à Oscilação de 3 a 9 dias. Além disso, aproximadamente $75 \%$ dos casos associados a este sistema durante os dois anos, também estão associados a um segundo sistema. Estas informações indicam que a Oscilação de 3 a 9 dias isoladamente, não parece exercer influência significativa sobre a precipitação do CLA, mas isso precisa ser confirmado em estudos futuros.

A maioria dos eventos associados a LI e SCM estão associados a ZCIT (Tabelas 3). Depois da ZCIT, o sistema que mais esteve associado aos SCM foi o VCAN. Em poucos casos, as LI estiveram associadas a ZCAS e Oscilação de 3 a 9 dias. Barbosa (2007) investigou as possíveis relações entre Oscilação de 3 a 9 dias e perturbações convectivas (PC), que têm uma estreita relação com LI de maior extensão espacial. Os resultados mostraram que a presença de Oscilação de 3 a 9 dias favorece a presença de PC confinadas (i.e., PC que se propaga até $150 \mathrm{~km}$ ) ou PC que se propagam para distâncias maiores do que $450 \mathrm{~km}$.

As principais características dos $\mathrm{SC}$ que atuaram no CLA durante 2005 e 2006 são mostradas na Figura 6. Um aspecto notável dos histogramas da Figura 6 é que, em geral, existe uma classe com número de eventos substancialmente maior que as outras classes. A Figura 6a mostra que de 60 a $80 \%$ dos eventos existem núcleos convectivos associados com

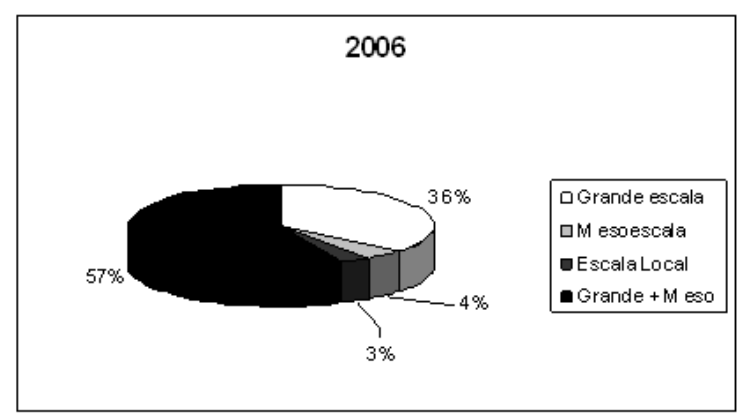

Figura 5 - Representação da quantidade de eventos de chuva (em porcentagem) ocorridos no CLA associados aos sistemas de grande, meso e pequena escala para o ano de (a) 2005 e (b) 2006. 
chuva sobre (ou próximo a) o CLA. O tamanho preferencial para os sistemas na região do CLA são os eventos inclusos na classe 1 da Figura 6b, que indica tamanho de nebulosidade dentro da mesoescala, i.e, sistemas que possuem a área entre $10^{4} \mathrm{~km}^{2}$ e $25 \times 10^{4} \mathrm{~km}^{2}$. A posição dos sistemas em relação ao CLA permanece preferencialmente na porção convectiva da nebulosidade, pois o maior número de eventos está alocado nas classes 1 (chuva na borda do sistema) e 2 (CLA no interior do sistema) da Figura 6c. A maior parte dos eventos apresenta intensificação por convecção diurna (classe 2 da Figura 6d) e por enfraquecimento/desintensificação (classe 0 da Figura 6d). Embora menor relativamente às outras classes, há um número substancial de sistemas que se intensificam por convecção noturna (classe 1 da Figura 6d). A situação atmosférica ao redor mostra que o sistema está geralmente incluso em um sistema maior (classe 2 da Figura 6e), e a origem preferencial dos sistemas é de sudeste e de nordeste (classes SE e NE da Figura 6f).

Dos eventos de precipitação ocorridos no CLA, aproximadamente $43 \%$ são associados a nebulosidade convectiva e $57 \%$ estão associados a nebulosidade estratiforme (Figura 7). Isso mostra a importância dos processos associados à nebulosidade estratiforme $(\mathrm{TB}>250 \mathrm{~K})$, não somente a convectiva, na produção de chuva no CLA. A formação da chuva associada à nebulosidade estratiforme se dá basicamente por dois processos.

Um grupo se forma por resquícios de um sistema formado anteriormente no CLA e que se encontra em fase de

Tabela 1 - Associação dos sistemas meteorológicos aos eventos de precipitação ocorridos no CLA nos anos de 2005 e 2006.

\begin{tabular}{|c|c|c|}
\hline Sistemas & $\mathbf{2 0 0 5}$ & $\mathbf{2 0 0 6}$ \\
\hline ZCIT & $54 \%$ & $79 \%$ \\
\hline LI & $20 \%$ & $46 \%$ \\
\hline VCAN & $15 \%$ & $22 \%$ \\
\hline ZCAS & $13 \%$ & $10 \%$ \\
\hline SCM & $25 \%$ & $26 \%$ \\
\hline 3a9 dias & $7 \%$ & $5 \%$ \\
\hline
\end{tabular}

Tabela 2 - Número de associações entre sistemas de grande escala (a) para o ano de 2005 e (b) para o ano de 2006.

\begin{tabular}{|c|c|c|c|c|}
\hline & ZCIT & ZCAS & VCAN & 3 a9 DIAS \\
\hline ZCIT & 87 & 24 & 23 & 10 \\
\hline ZCAS & $\times$ & 3 & 14 & 3 \\
\hline VCAN & $\times$ & $\times$ & 10 & 2 \\
\hline 3 a9 DIAS & $\times$ & $\times$ & $\times$ & 5 \\
\hline
\end{tabular}

(a)

\begin{tabular}{|c|c|c|c|c|}
\hline & ZCIT & ZCAS & VCAN & 3 a9 DIAS \\
\hline ZCIT & 163 & 23 & 27 & 9 \\
\hline ZCAS & $\times$ & 2 & 9 & 1 \\
\hline VCAN & $\times$ & $\times$ & 30 & 2 \\
\hline 3 a9 DIAS & $\times$ & $\times$ & $\times$ & 3 \\
\hline
\end{tabular}

(b) 
Tabela 3 - Associações entre sistemas de mesoescala com sistemas de grande escala (a) para o ano de 2005 e (b) para o ano de 2006.

\begin{tabular}{|c|c|c|c|c|c|}
\hline & ZCIT & ZCAS & VCAN & 3 a 9 DIAS & SOZIINHO \\
\hline LI & 31 & 9 & 11 & 7 & 12 \\
\hline SCM & 33 & 3 & 9 & 5 & 19 \\
\hline
\end{tabular}

(a)

\begin{tabular}{|c|c|c|c|c|c|}
\hline & ZCIT & ZCAS & VCAN & $\begin{array}{c}\text { 3 a 9 } \\
\text { DIAS }\end{array}$ & SOZINHO \\
\hline LI & 92 & 13 & 25 & 7 & 10 \\
\hline SCM & 66 & 10 & 11 & 4 & 2 \\
\hline
\end{tabular}

(b)

(a)

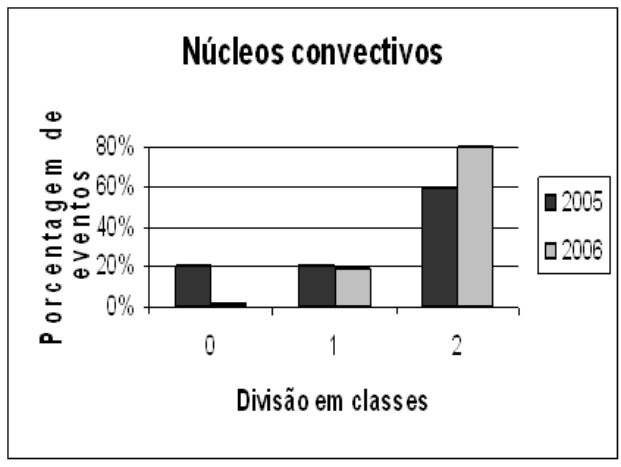

(c)

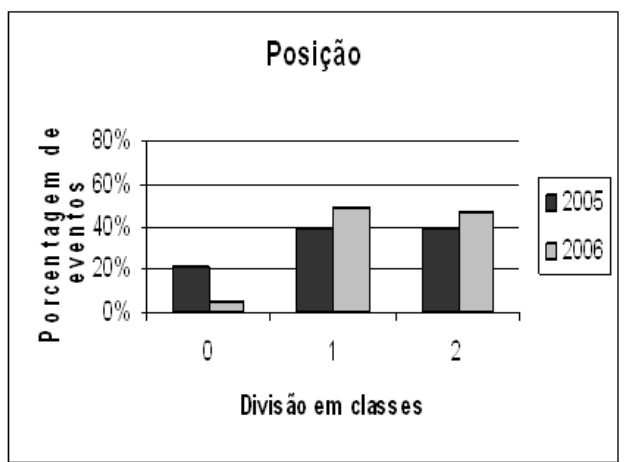

(e)

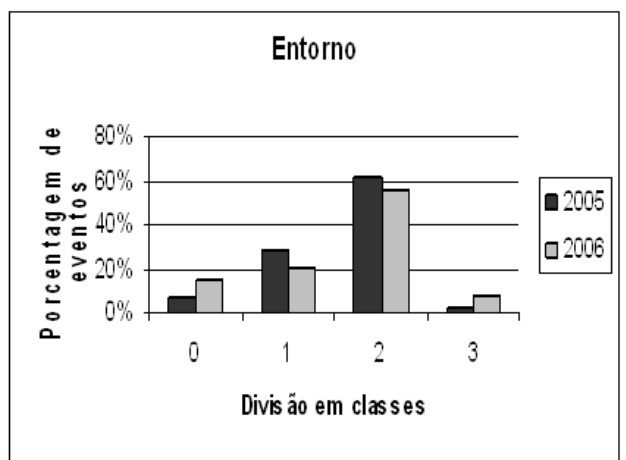

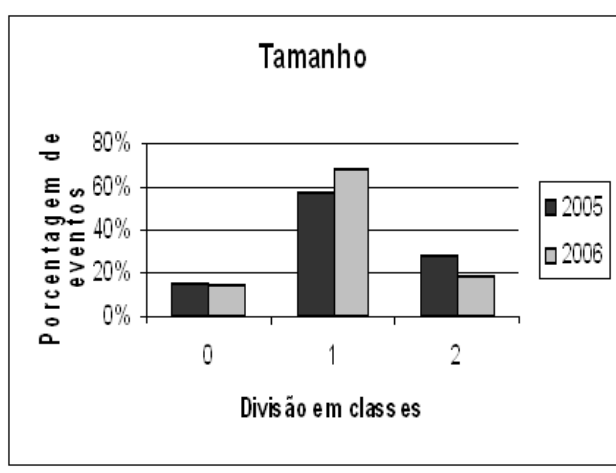

(b)

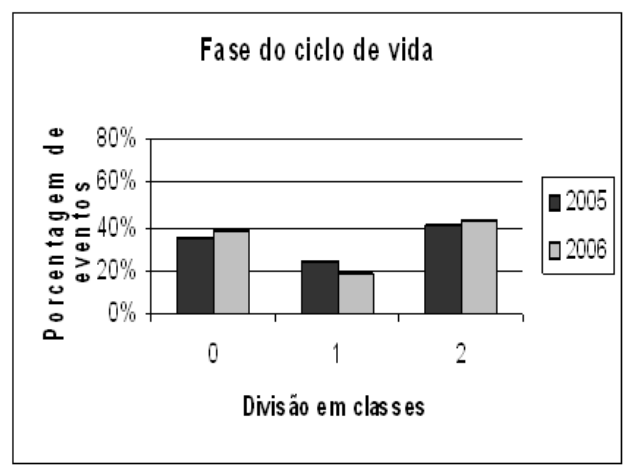

(d)

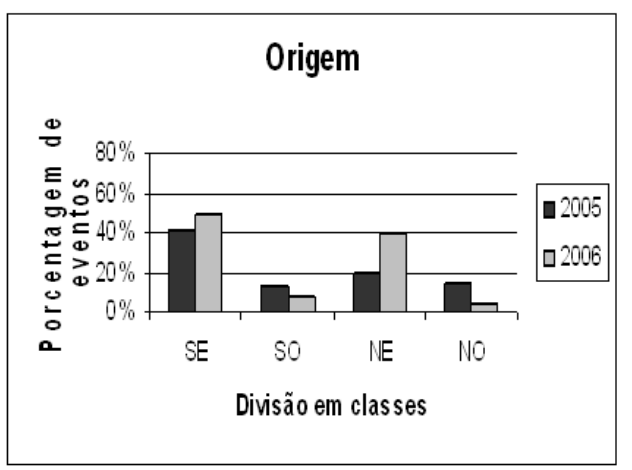

(f)

Figura 6 - Características gerais dos SC que atuaram no CLA durante 2005 (preto) e 2006 (cinza), com relação à porcentagem do número de eventos onde (a) mostra a classificação de núcleos convectivos, (b) tamanho, (c) posição do sistema com relação ao CLA, (d) fase do ciclo de vida, (e) entorno e (f) origem. 
desintensificação. Em 2005 (2006), a porcentagem destes eventos somou $52 \%$ (53\%) do total de eventos de nebulosidade estratiforme.

Um outro grupo se forma por uma nebulosidade que não está associada a nenhum tipo de enfraquecimento ou processo convectivo. A porcentagem deste grupo (em relação ao total de nebulosidade estratiforme) foi de $48 \%$ no ano de 2005 e $47 \%$ no ano de 2006.

Logo, cerca de um quarto do total de eventos que atuam no CLA não está associado a nenhum processo convectivo. Este resultado é importante, pois acredita-se que a distribuição da chuva na região equatorial é fortemente associada com a convecção profunda oriunda da ZCIT (Peixoto, 1992). Então, mesmo que fosse implementado um esquema perfeito de convecção profunda em modelo atmosférico, simulações sobre o CLA não representariam a ocorrência de precipitação em cerca de $1 / 4$ dos eventos.

Como muitos eventos apresentam características comuns, faz-se uma categorização. São propostas 4 categorias: eventos associados à ZCIT, ZCAS, aos sistemas de mesoescala ( $\mathrm{SCM}$ e LI) e nebulosidade isolada. O ciclo anual do número de eventos e quantidade de precipitação são apresentados para cada uma das categorias nas Figuras 8 - 11 .

Na categoria "ZCIT" (Figura 8a), o maior número de eventos se concentra no período chuvoso, e o menor número no período seco. Mais especificamente, o pico de eventos se dá nos meses de março e abril, meses em que a ZCIT encontra-se em sua posição mais austral segundo Lobo (1982). Os meses de janeiro e junho nitidamente se configuram como meses de transição. No período chuvoso, o total de precipitação associado com a ZCIT é maior do que nos meses adjacentes, com média de $250 \mathrm{~mm}$.mês ${ }^{-1}$, e um máximo de precipitação se apresenta em abril. O mês de dezembro apresenta um mínimo de precipitação de 27 mm.mês ${ }^{-1}$ (Figura 8b).

A Figura 9 apresenta características da categoria "ZCAS" e mostra que, durante o período de estudo, o período de atuação da ZCAS no CLA é em média de novembro a março. Um máximo de eventos e um máximo no total de precipitação coincidem exatamente no mês de março (Figura 9a-b).

O ciclo anual da categoria "LI" (Figura 10a) mostra que o mês de abril é o preferencial de atuação da LI, porém não é o

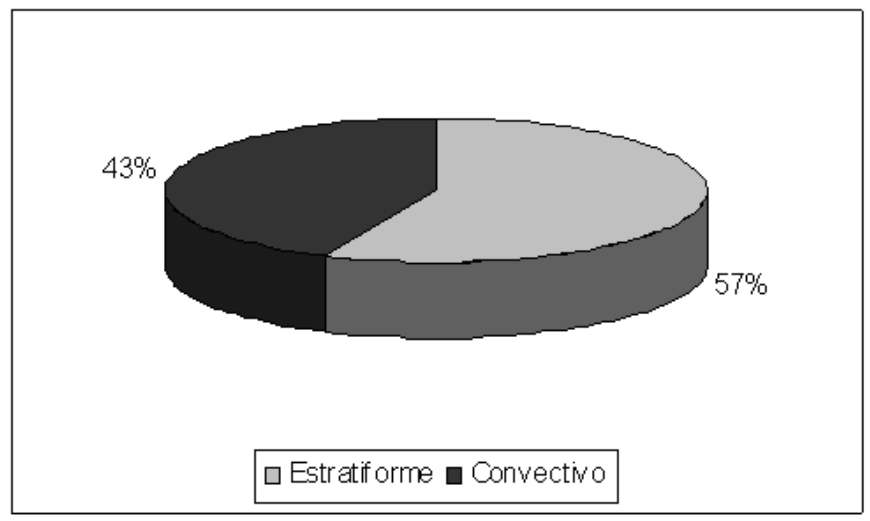

Figura 7 - Divisão dos eventos de chuva ocorridos em 2005 e 2006 no CLA em nebulosidade estratiforme e convectiva.
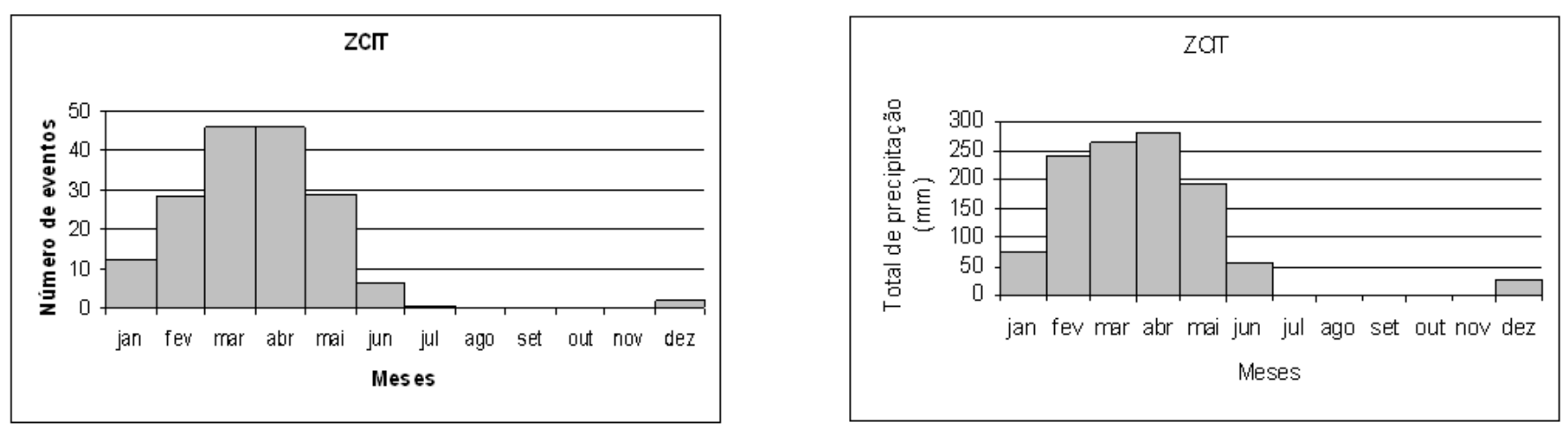

Figura 8 - Distribuição média mensal (a) do número de eventos por ano de precipitação no CLA (b) do total de precipitação no CLA associados com a ZCIT. 
mês com o maior total de precipitação gerada por este sistema (Figura 10b). Em abril, há um maior número de casos de ZCIT interagindo com a LI (não mostrado). Cohen et al. (1989), em sua climatologia feita para ocorrências de LI, observaram um máximo nos meses de abril e julho, e encontraram pouca influência de LI sobre a região amazônica durante a estação seca, concordando com os resultados apresentados na Figura 10a. A Figura 10b confirma a Figura 10a e mostra que, embora em junho o número de eventos seja menor, o total de precipitação é até mesmo superior ao do mês de abril. O total de precipitação é relativamente uniforme entre fevereiro e junho, sendo que os mínimos de precipitação ocorrem entre agosto e dezembro.

$\mathrm{Na}$ categoria "SCM", os eventos se distribuem de forma mais uniforme no decorrer do ano (Figura 10c) e concentram o maior número de eventos nos meses de março, abril e maio. $\mathrm{O}$ total de precipitação também se apresenta um tanto uniforme (Figura 10d), destacando-se, entretanto, o mês de fevereiro com um máximo de precipitação. O SCM não apresenta casos na estação seca, exceto em outubro.

As chuvas isoladas no CLA(Figura 11) se concentraram entre fevereiro e junho, acontecendo preferencialmente no mês de junho. É interessante ressaltar a ocorrência de uma queda abrupta do número de eventos de junho para julho (Figura 11a). AZCIT dificilmente está associada a chuvas isoladas no mês de junho, pois em junho a ZCIT se encontra em sua marcha para o Hemisfério Norte. O total de precipitação associado com a CI é basicamente uniforme apresentando um total maior (menor) no mês de junho (fevereiro). Nota-se que o mês de junho, mês com maior número de eventos (Figura 11a), apresenta o maior total de precipitação associado.

\section{CONCLUSÕES}

Este trabalho visou caracterizar a precipitação no Centro de Lançamento de Alcântara (CLA), onde é desenvolvido grande parte dos experimentos de interesse aeroespacial do Brasil. Segundo análises subjetivas de dados observados, foi possível avaliar a importância relativa dos sistemas meteorológicos que atuaram no CLA durante os anos 2005 e 2006. Além disso, buscou-se classificar os sistemas em pequena, média e grande escala e caracterizar os sistemas convectivos presentes na região quanto a sua morfologia e situação sinótica envolvida. Para tal, foram utilizados os seguintes conjuntos de dados: totais horários de precipitação no CLA, reanálises do NCEP, tempertura de brilho no canal infravermelho do satélite GOES - 12 e radiação de onda longa.

Os fatores de grande escala foram responsáveis por cerca de $40 \%$ do total de eventos chuvosos no CLA. Eles ainda interagiram com os fatores de mesoescala e somaram 26\% (57\%) do total de eventos em 2005 (2006), ratificando sua atuação como principal causador da chuva no CLA. O sistema meteorológico que esteve mais (menos) associado aos eventos de precipitação foi a ZCIT (Oscilação de 3 a 9 dias) com mais da metade (menos de $10 \%$ ) de eventos associados nos dois anos. Os casos de Oscilação de 3 a 9 dias individualmente parecem não exercer influência significativa sobre a precipitação do CLA, pois apresentaram poucos casos e geralmente estão associados a outro sistema. Os sistemas de mesoescala estudados (LI e SCM) se apresentaram na maioria dos casos em associação com a ZCIT.

Os eventos de precipitação foram divididos em dois grupos. O grupo no qual a nebulosidade esteve associada a processos convectivos e o outro formado por processos estratiformes. Os processos estratiformes se destacaram gerando cerca de $60 \%$ da precipitação ocorrida no CLA, enquanto que os processos convectivos geraram $40 \%$. Os sistemas formados por processos convectivos apresentaram algumas características, são elas: áreas entre $10^{4} \mathrm{~km}^{2}$ e $25 \times 10^{4} \mathrm{~km}^{2}$ (i.e., organização em mesoescala), a chuva está posicionada na porção convectiva do sistema, intensificam-se por convecção
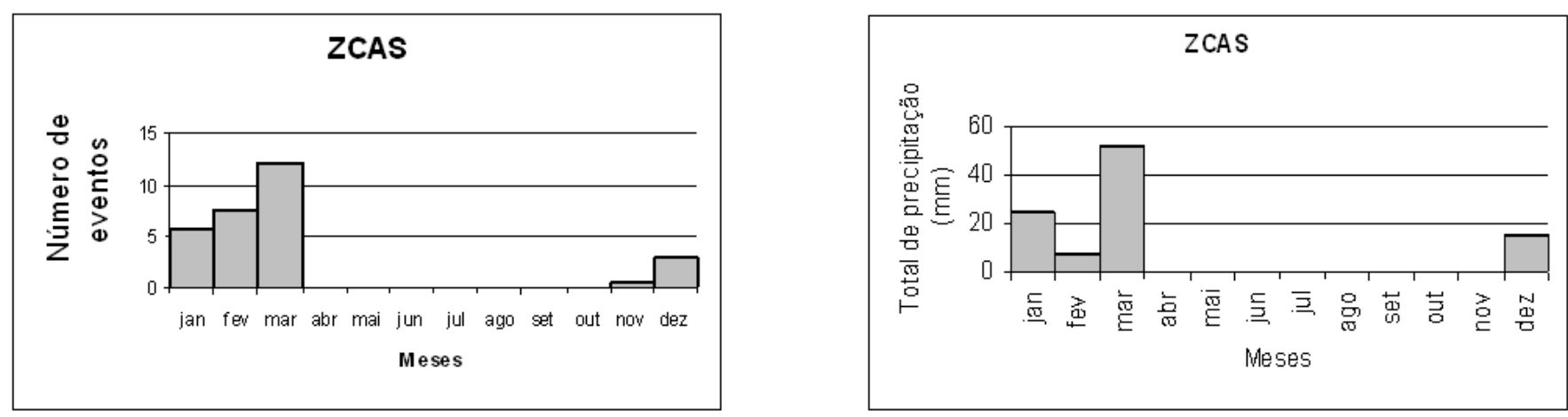

Figura 9 - Distribuição média mensal (a) do número de eventos por ano de precipitação no CLA (b) do total de precipitação no CLA associados com a ZCAS. 
diurna, encontram-se inclusos em um sistema de escala maior e a sua origem preferencial é de sudeste e de nordeste. Os sistemas formados por processos estratiformes estão quase que igualmente subdividos em outros dois grupos. No primeiro grupo encontram-se os sistemas que se formam por resquícios de outro formado anteriormente no CLA, e que se encontra em fase de desintensificação. No segundo grupo encontram-se os sistemas que se formam por uma nebulosidade, que não está associada a nenhum tipo de enfraquecimento ou processo convectivo.

Os eventos de chuva causados pela ZCIT apresentaram-se principalmente entre os meses de março e abril e concentraram
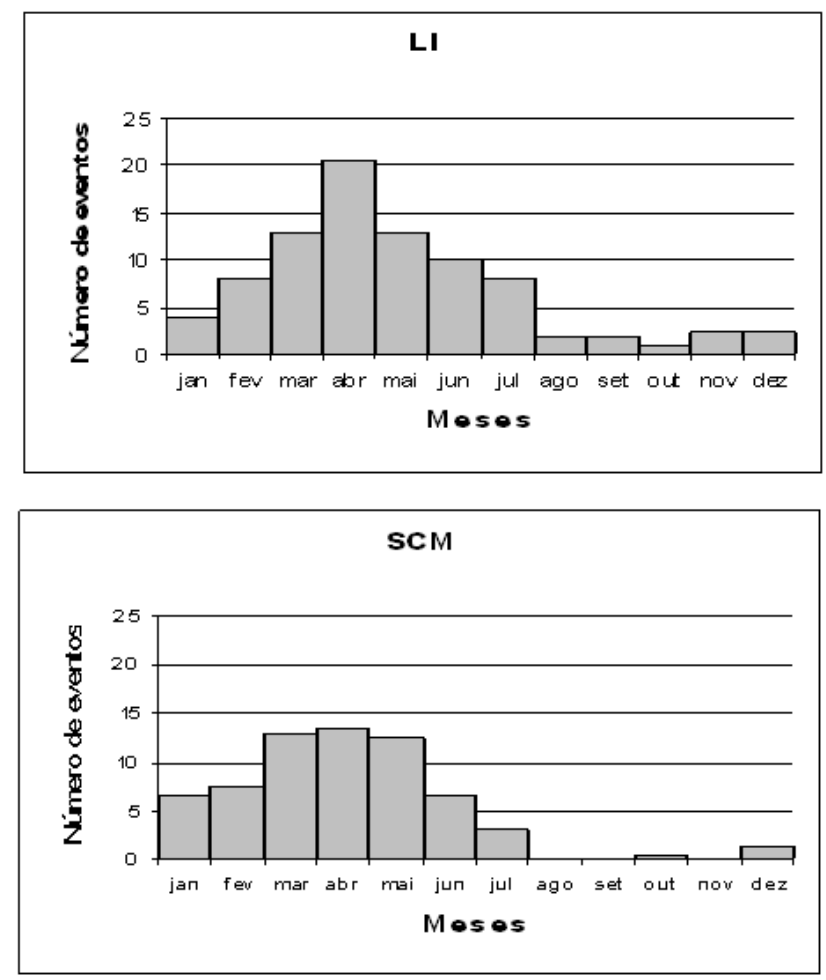

Figura 10 - Distribuição média mensal (a) do número de eventos por ano de precipitação no CLA e (b) do total de precipitação no CLA associados com LI (c) do número de eventos por ano de precipitação no CLA e (d) do total de precipitação no CLA associados com SCM.
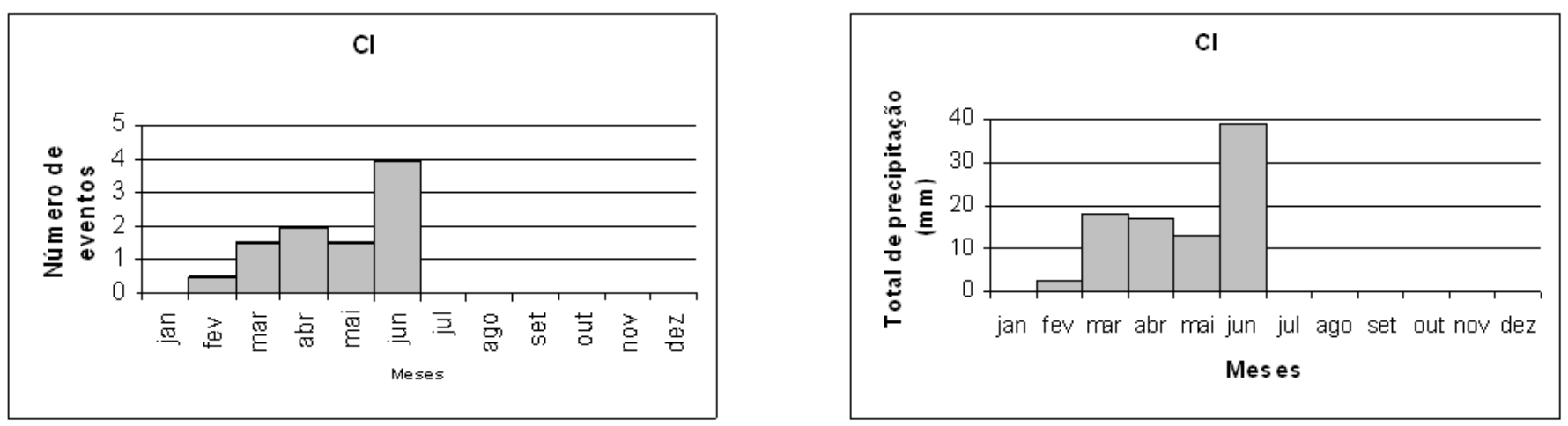

Figura 11 - Distribuição média mensal (a) do número de eventos por ano de precipitação no CLA e (b) do total de precipitação no CLA associados com CI. 


\section{AGRADECIMENTOS.}

Este artigo é parte do trabalho de mestrado da primeira autora sob orientação do segundo autor no Programa de Pós-Graduação em Meteorologia do Instituto Nacional de Pesquisas Espaciais (PGMET/INPE). Os autores agradecem à Coordenação de Aperfeiçoamento de Pessoal de Nível Superior (CAPES) pela concessão da bolsa de estudo da primeira autora.

\section{REFERÊNCIAS BIBLIOGRÁFICAS}

ALVES M. A. S.; MARQUES, R. F. C.; OYAMA, M. D. Detecção de distúrbios ondulatórios de leste sobre a região do centro de lançamento de Alcântara utilizando dados filtrados: estudo preliminar. In: CONGRESSO BRASILEIRO DE METEOROLOGIA, 15.,São Paulo, SP. Anais... São Paulo: SBMET, 2008.

BARBOSA, R. L. Interação das perturbações convectivas iniciadas na costa norte do Brasil com os distúrbios ondulatórios de leste. 151 p. (INPE-14433-TDI/1132). Dissertação (Mestrado em Meteorologia) - Instituto Nacional de Pesquisas Espaciais, São José dos Campos, 2007.

BARROS. S. S. Precipitação no Centro de Lançamento de Alcântara: aspectos observacionais e de modelagem. 102f. Dissertação (Mestrado em Meteorologia) -Instituto Nacional de Pesquisas Espaciais, São José dos Campos, 2008.

CAVALCANTI, I. F. A. Um estudo sobre interações entre sistemas de circulação de escala sinótica e circulações locais. 133 p. (INPE-2494-TDL/097). Dissertação (Mestrado em Meteorologia) - Instituto Nacional de Pesquisas Espaciais, São José dos Campos, 1982.

CLIMANALISE - Boletim de Monitoramento e Análise Climática. Cachoeira Paulista, SP: INPE, 2002, 2005 e 2006. Mensal. ISSN 0103-0019.

COHEN, J. C. P.; SILVA DIAS, M. F.; NOBRE, C. A. Aspectos climatológicos das linhas de instabilidade na Amazônia. Climanálise - Boletim de Monitoramento e Análise Climática, v. 4, n. 11, p. 34-40, nov. 1989. (INPE-6028PRE/2143).

FERREIRA, N. S. et al. Loczcit um procedimento numérico para localização do eixo central da zona de convergência intertropical no atlântico tropical. Revista Brasileira de Meteorologia, v. 20, n. 2, p. 159-164, 2005.

GUEDES, R. L.; MACHADO, L. A. T. Climatologia da estrutura vertical das perturbações convectivas sobre a América do Sul e adjacências. Revista Brasileira de Meteorologia, v. 18, n. 2, p. 215-230, dez 2003. (INPE-10653-PRE/6115).
KALNAY, E. et al. The NCEP/NCAR 40-year reanalysis project. Bulletin of American Meteorology Society, v. 77, p. 437-471, 1996.

LOBO, P. R. V. Um estudo climatológico da zona de convergência intertropical (ZCIT) e sua influencia sobre o nordeste do Brasil. 1982. 93 p. (INPE-2534-TDL/101). Dissertação (Mestrado em Meteorologia) - Instituto Nacional de Pesquisas Espaciais, São Jose dos Campos. 1982.

MADDOX, R. A. Mesoscale convective complexes. Bull. Amer. Meteor. Soc., 61, 1374-1387, 1980.

MARQUES, R. F. C., BAUNGARTNER, C. Estudo das variáveis meteorológicas associadas a posição da ZCIT do Atlântico, durante a estação chuvosa no Centro de Lançamento de Alcântara (CLA). In: CONGRESSO BRASILEIRO DE METEOROLOGIA, 15, São Paulo, SP. Anais... São Paulo: SBMET, 2008.

MARQUES, R. F. C.; FISCH, G. F. As atividades de meteorologia aeroespacial no Centro Técnico Aeroespacial (CTA). Boletim da Sociedade Brasileira de Meteorologia, v.29, n.3, p.21-25, 2005.

MOLION L. C. B.; BERNARDO, S. O. Uma revisão da dinâmica das chuvas no nordeste brasileiro. Revista Brasileira de Meteorologia, Rio de Janeiro (RJ), v. 17, n. 1, p. 1-10, 2002.

ORLANSKI, I. A rational subdivision of scales for atmospherics processes. Bull. Amer. Meteor. Soc. 56, p: 527-530, 1975.

PEIXOTO, J. P. Oort, A. H. Physics of climate. American Institute of Physics, 520p. 1992.

PEREIRA, E. I. et al. Atlas climatológico do Centro de Lançamento de Alcântara. São José dos Campos: Centro Técnico Aeroespacial. Relatório de desenvolvimento, ACA/ RT-01/01, GDO-000000/B0047. 2002.

SILVA, L. A. A influência do vórtice ciclônicos de altos níveis (VCAN) sobre a precipitação do nordeste do Brasil (NEB) e as características associadas. 134 p. (INPE14488-TDI/1169). Dissertação (Mestrado em Meteorologia) - Instituto Nacional de Pesquisas Espaciais, São José dos Campos, 2005.

TAVARES, P. S. Um estudo observacional da incursão de sistemas frontais no Nordeste Brasileiro e sua influência na atmosfera do Centro de Lançamento de Alcântara (CLA). Dissertação (Mestrado em Meteorologia) - Instituto Nacional de Pesquisas Espaciais, São José dos Campos, 152 p., 2008.

VILA, D. A.; MACHADO, L. A. T. Shape and radiative properties of convective systems observed from infrared satellite images. International Journal of Remote Sensing, v. 25, p. 1-16, 2004. (INPE-11148-PRE/6604). 